STUDIA EUROPAEA GNESNENSIA 16/2017

ISSN 2082-5951

DOI 10.14746/seg.2017.16.4

\author{
Ioan Piso \\ (Cluj-Napoca)
}

\title{
ZUR INSCHRIFT DES PRIMUS PILUS \\ G. BAIENIUS IANUARIUS AUS NOVAE ${ }^{\star}$
}

\begin{abstract}
Among the numerous inscriptions from Novae published by the Polish colleagues, that of G. Baienius Ianuarius deserves a special attention. It has been raised on October the $5^{\text {th }} 227 \mathrm{AD}$ under the governorship of Lucius Mantennius Sabinus. On the left side of the basis a text in hexameters contains the military carrier of C. Baienius Ianuarius. It is the lecture of this text that the author tries to improve. One of the conclusions is that G. Baienius Ianuarius carried out as a centurio of the legio I Italica a mission in Rome about 223/224 AD. Another conclusion is that the statue represented an aquila, symbol of the legion.
\end{abstract}

\section{Key words}

aquila, legio, legio I Adiutrix, legio II Adiutrix, centurio, L. Mantennius Sabinus, Novae, primus pilus, Severus Alexander

* Der vorliegende Aufsatz wurde im Rahmen eines Projektes des Rumänischen Ministeriums für Forschung und Innovation finanziert: CNCS - UEFISCDI, Projektnummer PN-III-P4-IDPCE-2016-0271.

Eine kritische Durchlesung des Textes verdanke ich Ekkehard Weber. 
Zunächst ist den polnischen Kollegen für ihre hervorragende Arbeit in Novae sowie für die tadellose Publikation der Ergebnisse $\mathrm{zu}$ danken. Besonders wichtig für die epigraphische Forschung sind die zwei Inschriftenbände, die 1992 und 1997 erschienen sind ${ }^{1}$. Ein großes Interesse erwecken jene Inschriften, die in den principia des Legionslagers gefunden wurden und die für den von der Legion in diesem Raum praktizierten Kult und für die diesbezügliche Rolle des primus pilus zeugen. Eines dieser Denkmäler hat mit Recht Aufmerksamkeit erregt. Es geht um die Statuenbasis, die an Iupiter Optimus Maximus Dolichenus für das Wohl des Kaisers Severus Alexander vom primus pilus G. Baienius Ianuarius am 5. Oktober 227 errichtet wurde. Ungewöhnlich am Text ist die Tatsache, dass er auf drei Seiten beschrieben wurde und besonders, dass eine dieser Seiten eine Versinschrift enthält. Im folgenden wird die gesamte Inschrift in der Form, in der sie publiziert wurde ${ }^{2}$, wiedergegeben:

Vordere Seite:

I(ovi) $O$ (ptimo) M(aximo)

Depulsori

[pr]o salute d(omini) n(ostri)

[[M(arci) Aurel(ii) Severi Ale-

5 xandri]] Pii Felicis

Aug(usti)

G(aius) Baienius G(ai) f(ilius) Clau-

dia [I]anuarius Cele-

ia p(rimus) [p(ilus)] leg(ionis) I Ital(icae) Seve-

10 rianae ex voto posu-

it.

Rechte Seite:

Dedic(atum) III Non(as) Oct(obres)

Albino et Maximo c[o(n)s(ulibus)]

per L(ucium) Mantennium Sa-

binum leg(atum) Aug(usti) pr(o) pr(aetore)

${ }^{1}$ J. Kolendo, Archeologia 19, 1968 (1969), S. 117-144; AE 1972, 526; P. Herz, ZPE 17, 1975, S. 196 (ohne die Versinschrift); ILBR 272; ILN 13; IGLN 25; J. Kolendo, ANRW II/18/2, 1989, S. 1064, Anm. 9, Nr. 1 (ohne die Versinschrift); idem, Mobilité sociale, S. 165 (die Versinschrift).

${ }^{2}$ Ad ILN 13 mit Abb. II; ad IGLN 25, mit S. 15, Abb. 4. 
5 et Servaeum Corne-

lianum leg(atum) leg(ionis).

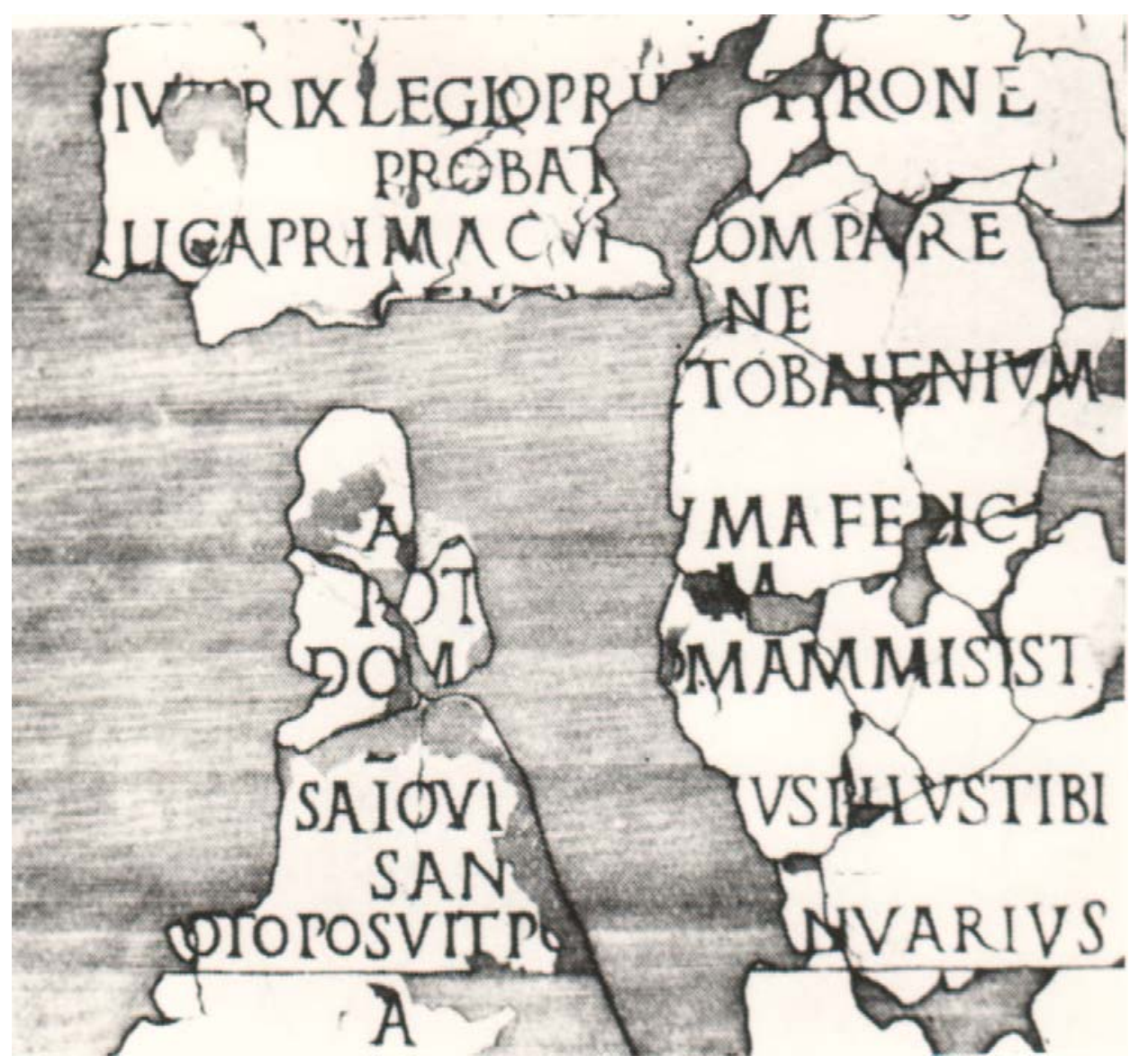

Abb. 1. Zeichnung nach ILN $13=$ IGLN 25

Linke Seite (Abb. 1):

[A]diutrix legio prim[a] tirone probat[o]

[-- - c]alica sive-aliga prima cu[m] compare cent[urio]ne

5 [- - - ]eto sive-cto Baienium [-- - ] 
[- - - ]AC[- - -]VMA felice

$[--] p o[-----] M$

[- - - ] DOM [- - R]omam misist[i]

10 [- - - $D, L$ sive $E$

[- - -]SA Iovi [- - prim]uspilus tibi

san[cto]

[ex v]oto posuit po[- - Ia]nuarius

a[ram?].

Die Statuenbasis wird hauptsächlich aufgrund der ausgezeichneten Kommentare von J. Kolendo behandelt, eines Wissenschaftlers und eines Kollegen, dessen Ableben wir alle zutiefst bedauern.

Die Inschrift wurde im Durchgang des westlichen Tores (porta principalis dextra) des Legionslagers zusammen mit fünf anderen Inschriften gefunden ${ }^{3}$. Das heißt, dass irgendwann um oder nach der Mitte des III. Jahrhunderts die Tore des Lagers vor einer drohenden großen Gefahr blockiert wurden ${ }^{4}$. Dem Inhalt nach stammt aber die Inschrift ganz bestimmt aus dem Hof der principia.

Dass es sich um eine Statuenbasis und nicht um eine Ara handelt, wird von den zwei Löchern bewiesen, die sich an der oberen Oberfläche des Denkmals befinden und zur Befestigung der Statue dienten ${ }^{5}$. Da im Text die aquila die als Symbol des Iupiter depulsor galt, nicht erwähnt wird, könnte vermutet werden, dass darauf wahrscheinlich die Statue eines Iupiter thronans oder fulgurator gesetzt war. Es wäre, wie wir weiter sehen werden, ein voreiliger Schluss.

Die Weihung wurde an Iupiter optimus maximus depulsor von G. Baienius G. f. Claudia Ianuarius aus Celeia, primus pilus der legio I Italica Severiana, für das Wohl des Kaisers Severus Alexander infolge eines Gelübdes ausgeführt. Die Auswahl eben dieser Gottheit ist durch die Herkunft des Dedikanten aus dem Municipium Celeia zu erklären, aus einer

\footnotetext{
${ }^{3} \mathrm{Zu}$ dieser Zeit wurden in mehreren Fällen die Tore der Legionslager und der Kastelle in Dakien blockiert; siehe D. Isac, [in:] XX Congresso Internacional de estudios sobre la frontera Romana. XX ${ }^{\text {th }}$ International Congress of Roman Frontier Studies, León (España), Septiembre, 2006 (Anejos de Gladius 13), Madrid 2009, S. 779-790; eine neue Datierung und Interpretation bei I. Piso, [in:] „Cities, Territories and Identities”. $1^{\text {st }}$ International Conference „Roman and Late Antique Thrace" (7-10 Octobr 2016, Plovdiv), Plovdiv 2017.

${ }^{4}$ Ad ILN 13; ad IGLN 25.

${ }^{5}$ Ad ILN 13; ad IGLN 25.
} 
Gegend also, wo diese Jupitervariante zu Hause war ${ }^{6}$. Nach dem Text wurde das Denkmal von dem Statthalter L. Mantennius Sabinus ${ }^{7}$ und von dem Legionslegaten Servaeus Cornelianus ${ }^{8}$ geweiht. Es ist anzunehmen, dass die Weihung in der Tat seitens des Legionslegaten unter der Autorität des L. Mantennius Sabinus ausgeführt wurde ${ }^{9}$. Die Weihung ist eindeutig auf den 5. Oktober 227 datiert. P. Herz meinte, dass dieses Datum eine Beziehung zum natalis aquilae hätte, weil daselbst ein anderes Denkmal dis militaribus, genio, virtuti, aquilae sanc(tae) signisque leg(ionis) I Ital(icae) Severianae, an einem nahen Tag, am 20. September $(224)^{10}$ geweiht wurde. Das ist gut möglich, aber der 20. September und der 5. Oktober sind doch nicht so nahe beisammen. Um eine endgültige Meinung darüber zu gewinnen, soll man geduldig auf die Lesung und die Interpretation der Versinschrift warten. Diese habe ich folgenderweise gelesen (Abb. 2):

[A]diutrix legio prim [o?] tirone

probat[o]

[In c]aliga sive [It]alica prima cum compare

centu[rio]ne

5 [- - fa]cto Baienium

$[---]$

[- - It]al[ica pr]ima felice

pot[ente]m

[Ex iussu] dom[ini R]omam misist[i]

10 e[undem]

[Impen]sa Iovi [sua prim]us pilus tibi

san[cto]

[EX v]oto posuit po[rtis Ia]nuarius

a[ram?].

\footnotetext{
${ }^{6}$ Für die norisch-pannonische Herkunft des Iupiter depulsor siehe besonders H.-G. Pflaum, AIPhO (= Mélanges Isidore Lévy), S. 445-460; für diesen besprochenen Fall siehe J. Kolendo, ANRW II/18/2, 1989, S. 1071

${ }^{7}$ Siehe für L. Mantennius Sabinus L. Petersen, PIR² M 172; W. Eck, RE Suppl. XIV, 1974, 274, no 3; B.E. Thomasson LP I, 142 Nr. 121; idem, LP I2. Er ist in Untermoesien auch durch die Inschriften AE 1922, 73 = ISM V 57 (Ulmetum), AE 1960, 349 = ISM V 8 (Capidava) und IGBR I² 70 bis (Odessos) bekannt.

${ }^{8}$ Siehe für Q. Servaeus Fuscus Cornelianus I. Piso, Fasti I, S. 257-260; K. Wachtel, PIR ${ }^{2}$ S 560.

${ }^{9}$ Vgl. CIL III 1092 = IDR III/5, 235 (Apulum): - - - d(ono) d(edit) sub Vespron(io) [Can]dido co(n)s(ulari) dedic[ante C]aerellio Sabi[n]o l[eg(ato)].

${ }^{10}$ CIL III $6224=7591=$ ILS $2295=$ AE 1966, $355=$ ILBR $282=$ IGLN 12.
} 


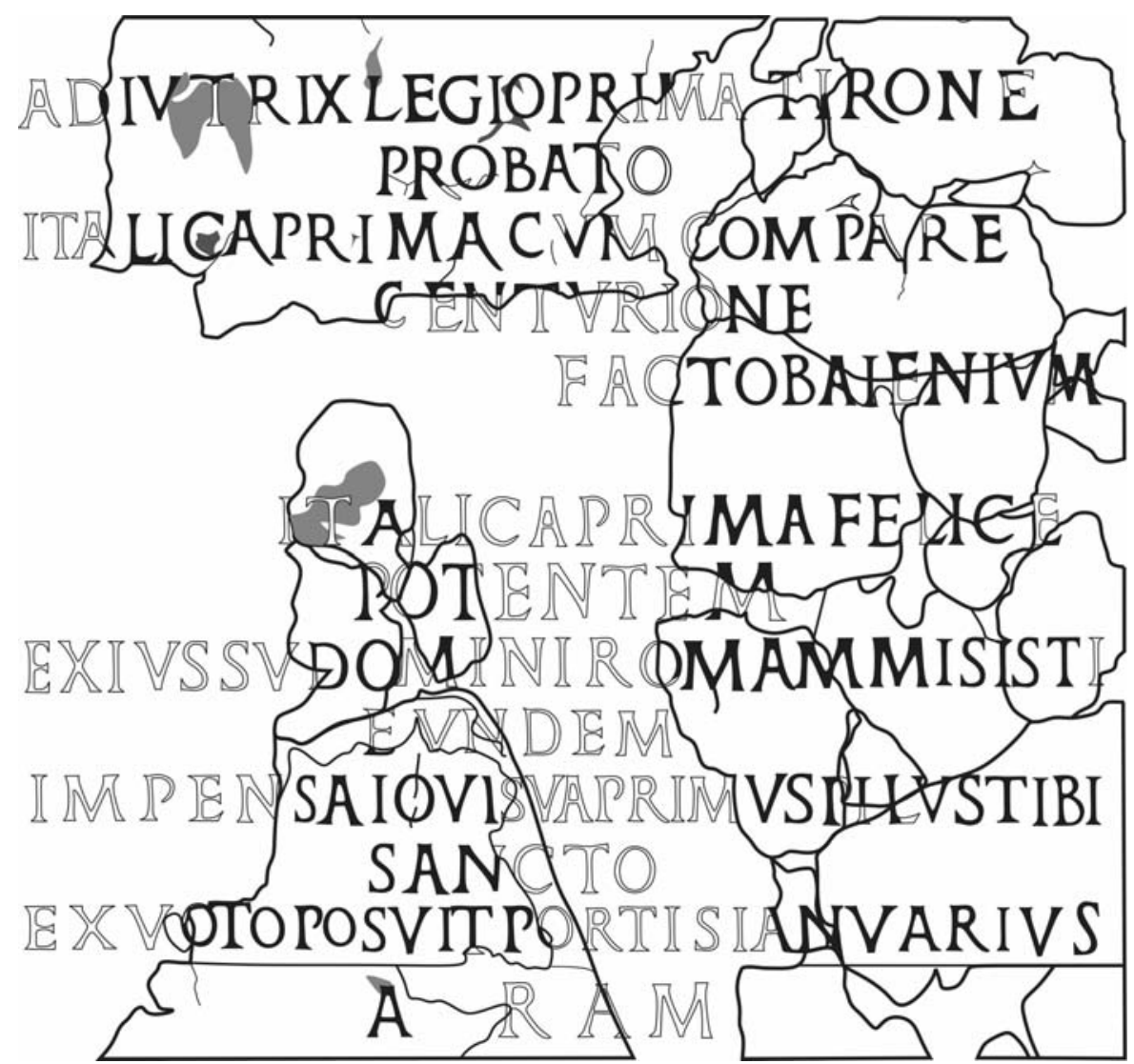

Abb. 2. Neue Lesung

Ganz richtig bemerkte J. Kolendo, dass das Gedicht einen biographischen Charakter hat. Es ist zuerst hinzuzufügen, dass der primus pilus in der dritten Person erscheint und zweitens, dass er mit jemandem einen Dialog führt. Mit wem, dass wird sich am Ende herausstellen. Es ist zu bemerken, dass das Gedicht in Hexametern verfasst wurde. Nützlich für die Rekonstitution des Textes ist, dass die Längen der Silben, mit Ausnahme von compāre (Z.3) und vielleicht von prima (Z. 1) richtig eingehalten wurden.

\section{Z. 1-2: [A]diutrix legio prim [o?] tirone / probat[o]}

„Die legio Adiutrix zuerst, nach der Prüfung des Rekruten...”. Dem auf die Legion bezüglichen Zahlwort prim[a] würde ich das Adverb prim[o] oder prim[um] vorziehen, denn primā, mit langem a, wäre im Ablativ, während Adiutrix legio im Nominativ steht. Wie oben bemerkt, kann es sich auch hier 
um ein Irrtum des Dichters handeln. Falls wir primo oder primum lesen, bezieht sich dieses als Adverb auf tirone probato und wir können nicht wissen, ob es sich um die Ioder II Adiutrix handelt ${ }^{11}$. Es fehlt das Prädikat. Es dürfte sich in den Lücken der Z. 5-6 befunden haben, was heißt, dass die Adiutrix legio weiterhin als Subjekt besteht. Einen Vorschlag zur Ergänzung dieses Prädikats traue ich mich nicht zu machen.

\section{Z. 3/4: [In c]aliga prima cum compare centu[rio]ne}

„Am Anfang der militärischen Dienstzeit, mit einem gleichaltrigen Centurio..." In caliga prima befindet sich ein Soldat nach seiner Prüfung als Rekrut (probatio), in der ersten Periode seines Militärdienstes ${ }^{12}$. Unter dem compar centurio hat man bisher einen Mann verstanden, der gleichzeitig mit G. Baienius Ianuarius rekrutiert wurde ${ }^{13}$ und mit ihm nicht nur zum Centurionat emporgekommen ist, sondern auch in die legio I Italica verlegt wurde. Kann man in caliga prima auch einen compar centurio haben? In caliga militare versteht man gewöhnlich, den Militärdienst als gemeiner Soldat auszuüben ${ }^{14}$. Man kennt aber auch den Fall des C. Memmius Iulius Maioriarius, dessen militärische Laufbahn sich folgendermassen entwickelte: caliga prima optioni primipilariorum subcommentar(i)ensi comment[ar](i)ensi corniculario pr(a)ef(ectorum) praetorio primipilari v(iro) e(gregio) ducenario ${ }^{15}$, wobei sich caligā primā im Ablativ befindet. Man kann daraus eher verstehen, dass er das Amt eines optio primipilariorum, im Rahmen der caliga prima bekleidete ${ }^{16}$. Demzufolge ist durch caliga prima jeder Anfang einer militärischen Laufbahn und nicht nur die untersten Ämter zu verstehen. Dann dürften G. Baienius Ianuarius und sein Kollege aus dem Ritterstand (ex equite Romano) direkt als centuriones rekrutiert worden $\operatorname{sein}^{17}$ und somit bekleideten sie den ersten Centurionenposten in caliga prima. Aus Raumgründen oder falls man die erste Erklärung nicht akzeptiert, besteht auch die Möglichkeit, [It]alica prima zu ergänzen, was für den ersten Teil der Laufbahn verschiedenartige Perspektiven öffnet.

\footnotetext{
${ }^{11}$ Bisher galt die legio I Adiutrix als sicher.

${ }^{12}$ J. Kolendo, Mobilité sociale, S. 166.

${ }^{13}$ Ebenda: compagnon du centurion.

${ }^{14}$ Siehe CIL III 7108 = ILS 2149 = IK 23, 475 (Smyrna); CIL VI $2440=$ ILS 2077.

${ }^{15}$ A. Łajtar, J. Żelazowski, ZPE 192, 2014, S. 273-281 = AE 2014, 1031 a-c (Scodra).

${ }^{16}$ A. Łajtar und J. Żelazowski (a.a.O. 275) begnügten sich damit, diese Möglichkeit zu erwähnen, ohne sie zu verfolgen.

${ }_{17}$ Siehe für solche Fälle H. Zwicky, Zur Verwendung des Militärs in der Verwaltung der römischen Kaiserzeit, Zürich 1944, S. 90-93; B. Dobson, apud A. v. Domaszewski, Rangordnung², S. XXI.
} 


\section{Z. 5-6: [- - - fa]c to Baienium / [- - -]}

Das Prädikat des ersten Satzes dürfte sich in der erste Hälfte der. Z. 5 oder in der Z. 6, das heißt im letzten Fuß des Hexameters, befinden. [Fa]cto gehört $\mathrm{zu}$ einem Ablativus absolutus, der eine Errungenschaft oder eine Beförderung ausdrückte. Baienium aus der Z. 5 ist mit pot[ente]m aus der Z. 8 zu verbinden.

Z. 7-10: [- - - It]al[ica pr]ima felice / pot[ente]m / [ex iussu] dom[ini RJomam misist[i]/e[undem].

„... in der glücklichen ersten Legion ... den mächtigen Baienius; (dem Befehl) des Herrn (folgend) hast Du denselben nach Rom geschickt". Aus der lückenhaften Z. 7 versteht man, dass sich Baienius Ianuarius schon in der legio I Italica befindet. Zu bemerken ist es, dass in Italica das I im Prinzip kurz ist, dass aber in Gedichten das Wort gelegentlich daktylisch gemessen wird $^{18}$. Die legio I Italica trug offiziell weder das Epitheton felix, noch andere Epitheta $^{19}$, mit Ausnahme der Kaiserepitheta. Dann ist hier felice ein von Ianuarius gewählte Attribut und heißt einfach „vom Glück begünstigt”. In Z. 9, statt ex iussu kann auch mandato oder praecepto stehen. Unter dem dominus versteht man selbstverständlich den Kaiser.

Z. 11-14: [Impen]sa Iovi [sua prim]us pilus tibi / san[cto / ex v]oto posuit po[rtis Ia]nuarius / a[ram?].

"Auf seinen (eigenen) Kosten errichtete der primus pilus Ianuarius bei den Toren (den Eingängen), infolge eines Gelübdes, eine Ara an Iupiter, der Dir heilig ist". In Z. 13: po[rta] oder po[rtis], aber der Plural mit dem Richtungsdativ scheint für eine Dichtung geeigneter zu sein. Gemeint sind wahrscheinlich die Eingänge, die vom Hof der principia in die basilica führen ${ }^{20}$. J. Kolendo meinte, dass die Z. 11-14 eine Invokation an Iupiter ausdrücken $^{21}$. Der Dialog, vom Verbum misisti (Z. 9) und vom Pronomen tibi (Z. 11) angedeutet, wird aber keineswegs mit Iupiter geführt. Wer hätte

\footnotetext{
${ }^{18}$ Siehe Oxford Latin Dictionary, Oxford 1968, S. 974: „first syll. naturally short (Quint. Inst. 1, 5,18 ) and so scanned in Lucil. 825, but in dactylic verse lengthened metri gratia.

${ }^{19}$ Siehe, zum Beispiel, die Typologie der Ziegestempel der Legion, bei T. Sarnowski, Archeologia Warszawa 34, 1983, S. 17-61; N. Gudea, EphNap 13, 2003, S. 195-213. Die Behauptung von M. Absil (in: Légions I, S. 231), dass in der Inschrift CIL III 13272 die legio I Italica die Epitheta felix, victrix, pia trägt, beruht auf einem Irrtum.

${ }^{20}$ Siehe den Plan in IGLN S. 20, Abb. 5; T. Sarnowski, Roman Frontier Studies 1989, S. 303-307; idem, Archeologia Warszawa 56, 2005, S. 141-152, Abb. 3.

${ }^{21}$ J. Kolendo, Mobilité sociale, S. 166.
} 
Ianuarius nach Rom schicken können? Die Legion? Das ist unmöglich, denn die Legion ist keine obere Gewalt, die Befehle geben kann. Außerdem befindet sich der Name der Legion im Ablativ und nicht im Nominativ. Noch weniger geht es um den Legionslegaten oder um den Statthalter, denn weder sie, noch ihre Funktion werden erwähnt. Wen spricht denn Ianuarius an? Nur ein Wesen, das deswegen nicht genannt wird, weil es als Statue leicht erkennbar war. Meiner Ansicht nach wurde das Gespräch zwischen dem primus pilus und der aquila geführt, die auf der Statuenbasis dargestellt war. Die aquila bedeutete für die Legion viel mehr als ein Symbol, sie war der Band, der die Legion, die das römische Volk vertrat, Jupiter, den Schirmherr eben des römischen Volkes, und den Imperator, dem die auspicia maxima zustanden, vereinigte. Eben der aquila war Iupiter heilig (Z. 11-12). Die aquila war es auch, gemäß dem Geist der römischen Staatsreligion, die Ianuarius nach Rom geschickt hat, während der Legionslegat und der Statthalter nur Schattenfiguren in diesem staatsrechtlichen Gewebe darstellten.

Es handelt sich in unserem Fall bestimmt nicht um die goldene aquila, die in der aedes principiorum aufbewahrt war $^{22}$ und sich unter der Aufsicht des primus pilus befand ${ }^{23}$, sondern um eine silberne oder versilberte Kopie davon, wie im Falle von Apulum ${ }^{24}$. Aus den principia von Novae kennt man ein Denkmal, das dis militaribus, genio, virtuti, aquilae sanc(tae) signisque leg(ionis) I Ital(icae) Severianae von einem primus pilus errichtet wurde ${ }^{25}$, ohne das wir wissen, wen von diesen Gottheiten die Statue darstellte. Daselbst wurden zwei weitere Denkmäler verschiedenen Gottheiten zur Ehre der aquila errichtet, wahrscheinlich ohne dass diese selbst dargestellt worden wäre ${ }^{26}$.

Es stellt sich die Frage, warum Ianuarius von einer Ara spricht (Z. 14), wenn das Denkmal eindeutig eine Statuenbasis ist. Entweder ist das eine poetische Lizenz, oder hat Ianuarius vor den Eingängen auch eine Ara gestellt.

Aus der Versinschrift dringen einige wichtigen Ereignisse aus der militärischen Laufbahn des G. Baienius Ianuarius durch. Als einfacher

\footnotetext{
${ }^{22}$ Dio $40,18,1$.

${ }^{23}$ Vegetius II 8: centurio primi pili, qui - - aquilae praeerat.

${ }^{24}$ AE 2007, 1199: L(ucius) Aninius L(ucii) f(ilius) | Pap(iria) Firminus | Tridente equo | publ(ico) ex (trecenario) |5 p(rimus) p(ilus) | leg(ionis) XIII Gem(inae) | tetrastylum fecit | et aquilam $\mid{ }^{10}$ argenteam | posuit; siehe V. Moga, I. Piso, M. Drîmbărean, AMN 43-44, 2006-2007, S. 177-184.

${ }^{25}$ CIL III $6224=7591=$ ILBG $282=$ IGLN 12.

${ }^{26}$ AE 1988, $984=$ ILBG $282=$ ILN $18=$ IGLN 33; AE 1982, $849=$ ILBG 268ter = ILN $28=$ IGLN 47; für Denkmäler, die honori aquilae gestzt wurden, siehe P. Herz, ZPE 17, 1975, S. 183-189.
} 
Rekrut $^{27}$ wurde er wahrscheinlich in der legio I Adiutrix aus Brigetio, falls nicht in der legio II Adiutrix aus Aquincum, geprüft (tirone probato) ${ }^{28}$. Falls er mit ungefähr fünfzig Jahren den Primipilat erreichte ${ }^{29}$, dürfte er um 200 rekrutiert worden sein. Jetzt begann das langwierige Emporkommen zur Stelle eines hohen Principalis, bevor er in derselben Legion zum Centurio ernannt wurde ${ }^{30}$. Von jetzt an avancierte er als Centurio in zwei Richtungen: von der acies der hastati zu jener der pili und von der zehnten zur ersten Cohors $^{31}$. Diese Beförderung geschah zumeist in mehreren Legionen, je nachdem freie Plätze vorhanden waren ${ }^{32}$. Man pflegte zuerst innerhalb der Legionen derselben Provinz zu promovieren, um weiter in Legionen einer anderen Provinz oder einer anderen Armeegruppe die Laufbahn fortzusetzen. Dieses System der Centurionenbeförderung hatte auch den Zweck, das gesamte römische Heer taktisch und als Disziplin zu vereinheitlichen. In seiner Versinschrift erwähnte Ianuarius nur die Legion in welcher er den ersten Centurionat erreichte, eine legio Adiutrix, und jene, in der er zum Primipilat befördert wurde, die legio I Italica, nicht aber auch die wahrscheinlichen Zwischenstationen. In die legio I Italica kam er also als hochrangiger Centurio. Als solcher und nicht als primus pilus wurde er auf Befehl des Kaisers mit einer Aufgabe nach Rom geschickt ${ }^{33}$. Auf keinen Fall durfte er als primus pilus seine Legion verlassen ${ }^{34}$. Worum es ging, können wir nur vermuten. Laut einer Interpretation von P. Oxy. 2565, fand die Ermordung in Rom des Prätorianerpräfekten Domitius Ulpianus seitens der

\footnotetext{
${ }^{27}$ J. Kolendo, Mobilité sociale, S. 166; siehe auch AE 1985, 735 = ILN 27 = IGLN 46: - - - quod tiro aput leg(ionem) XX V(aleriam) V(ictricem) voveram nunc p(rimus) p(ilus) leg(ionis) I Ital(icae) stip(endiorum) LVII $v$ (otum) s(olvi) - - -

${ }^{28}$ Siehe Vegetius I 1-27; dazu Fr. Lammert, RE VI A2, 1937, 1448-1450; P. Herz, ZPE 17, 1975, S. 190; R. Davies, Service 3-18.

${ }^{29}$ B. Dobson, Primipilares 16, S. 60-62; G. Cupcea, Professional Ranks 116. Vgl. aber den L. Maximius L. f. Voltiniae Gaetulicus Viennae (AE 1985, 735 = ILN 27 = IGLN 46), der als primus pilus in Novae ungefähr 74-77 Jahre alt war; siehe L. Mrozewicz, ZPE 57, 1984, S. 181-188; idem, Eos 74, 1986, S. 303-308.

${ }^{30}$ E. Birley, Laureae Aquincenses II 1941, S. 48 = idem, Roman Army, S. 190; B. Dobson, Primipilares, S. 40-59.

${ }^{31}$ Siehe I.-A. Petiș, AMN 41-42, 2004-2005, S. 125-127 mit Abb. 1.

${ }^{32}$ A. v. Domaszewski, Rangordnung², S. 90-97; E. Birley, CarnuntumJb 1963/1964, S. 21-33 = idem, Roman Army, S. 206-220.

${ }^{33}$ Laut J. Kolendo (Mobilité sociale, S. 166) wurde Ianuarius nach Rom versetzt. Darum ging es aber wohl nicht.

${ }^{34}$ Ein Drittel der legio V Macedonica ist in Caracallas parthischen Krieg gezogen. Die primi ordines, einschließlich des primus pilus, sind aber in Potaissa geblieben, I. Piso, in: Ad fines, S. 81-88.
} 
Prätorianer in den Jahren 223-224 statt ${ }^{35}$. Die Freveltat wurde sogar vor den Augen des Kaisers und seiner Mutter begangen ${ }^{36}$. Es steht zu erwarten, dass die beiden daraufhin einige Maßnahmen getroffen haben. Es ist wohl möglich, dass Legionsvexillationen von der Donau nach Rom beordert wurden, um den Übermut der Prätorianer zu dämpfen, und dass der Centurio Ianuarius sich an dieser Mission beteiligte. Falls es so ist, könnte Ianuarius die Reise nach Rom 224/225 unternommen haben.

Die Idee, dass der erste Primipilat auch in der Prinzipatszeit nur ein Jahr dauerte, ist sehr verbreitet ${ }^{37}$. Dafür gibt es, laut P. Herz, keine triftigen Argumente $^{38}$ und, andererseits kann man, was die Dauer betrifft, den Primipilat nicht mit irgendeiner Magistratur vergleichen.

Die Inschrift des G. Baienius Ianuarius gibt uns den Anlass, in das militärische Wesen und in die römische Staatsreligion einen tiefen Blick zu werfen. Hier, wie auch anderswo, trifft das Wort von Peter Herz zu: „Wir haben hier einen weiteren Beweis für das Faktum vor uns, dass in der Antike religiöse und offiziell-administrative Aufgaben in wesentlich größerem Umfang miteinander verflochten waren, als man es sich heute vorstellen kann"39.

\section{ABOUT THE INSCRIPTION OF THE PRIMUS PILUS G. BAIENIUS IANUARIUS FROM NOVAE}

\section{Summary}

A new lecture has been proposed for the text in hexameters of the well known inscription of the primus pilus $\mathrm{G}$. Baienius Ianuarius from Novae, dated on October the $5^{\text {th }} 227 \mathrm{AD}$. This text contains in a poetic form the carreer of the officer.

\footnotetext{
${ }^{35}$ P. Oxy, ad 2565; siehe F. Millar, A Study of Cassius Dio, Oxford 1964, S. 24; P. Herz, ZPE 17, 1975, S. 187-188.

${ }^{36}$ Die Quellen dazu: Dio (Xiph.) 80, 2, 2-4; Zonaras 12, 15; Zosimos 1, 11, 3; Syncell. Chron. 1, 673; siehe dazu A. Stein, PIR² D 169.

${ }^{37}$ B. Dobson, Primipilares, S. 60; L. Keppie, Roman Army, S. 179; G. Cupcea, Professional Ranks, S. 115. Laut A. Dobson (Primipilares, S. 60) wurden solche Inschriften zum Ende der Amtszeit des primus pilus gesetzt. Sie konnten aber ebenso gut auch am natalis aquilae gesetzt werden (P. Herz, ZPE 17, 1975, S. 185-193).

${ }^{38}$ P. Herz, ZPE 17, 1975, S. 191.

${ }^{39}$ Ibidem, S. 190.
} 


\section{Bibliographie}

Ad fines $=$ A. Tomas (Hg.), Ad fines imperii Romani. Studia Thaddaeo Sarnowski septuagenario $\mathrm{ab}$ amicis, collegis discipulisque dedicata, Varsaviae 2015.

Birley E., Promotions and transfers in the Roman Army. II: Centurionate, Carnuntum Jahrbuch 8, 1963/1964, S. 21-33 = idem, Roman Army, S. 206-220.

Birley E., Roman Army = idem, Roman Army. Papers 1929-1986, Amsterdam 1988.

Birley E., The origins of legionary centurions, Laureae Aquincenses II 1941, 47-62 = idem, Roman Army, S. 189-205.

Cities, Territories and Identities. $1^{\text {st }}$ International Conference Roman and Late Antique Thrace (7-10 October 2016, Plovdiv), Plovdiv 2017.

Cupcea G., Professional Ranks = Professional Ranks in the Roman Army of Dacia, BAR International Series 2681, Oxford 2014.

Davies R., Service = Service in the Roman Army, Edinburgh 1989.

Dobson B., Primipilares = Die Primipilares. Entwicklung und Bedeutung, Laufbahnen und Persönlichkeiten eines römischen Offiziersranges, Köln-Wien 1978.

Domaszewski A. v., Rangordnung ${ }^{2}=$ Die Rangordnung des römischen Heeres ${ }^{2}$, Köln-Graz 1967.

Gudea N., Contribuţii la istoria militară a provinciei Moesia inferior. 2. Pentru o nouă tipologie a stampilor legiunii I Italica, Ephemeris Napocensis 13, 2003, S. 195-213.

Herz P., Honos Aquilae, Zeitschrift für Papyrologie und Epigraphik 17, 1975, S. 181-197.

IGLN = J. Kolendo, V. Božilova, A. Bresson, Th. Drew-Bear, Inscriptions grecques et latines de Novae (Mésie Inférieure), Bordeaux 1997.

ILBR = B. Gerov, Inscriptiones Latinae in Bulgaria repertae, Serdicae 1989.

ILN = V. Božilova, J. Kolendo, L. Mrozewicz, Inscriptions latines de Novae, Poznań 1992.

Isac D., Repairing works and reconstructions on the limes Dacicus in the late $3^{\text {rd }}$ Century AD, [in:] XX Congresso Internacional de estudios sobre la frontera Romana. $X^{\text {th }}$ International Congress of Roman Frontier Studies, León (España), Septiembre, 2006 (Anejos de Gladius 13), Madrid 2009, S. 779-790.

Keppie L., Roman Army = The Making of the Roman Army. From Republic to Empire, London 1984.

Kolendo J., Inscription de l'an 227 en l'honneur de Jupiter Depulsor découverte à Novae, Archeologia 19, 1968 (1969), S. 117-144.

Kolendo J., Le culte de Jupiter Depulsor et les incursions des Barbares, ANRW II 18.2, 1989, S. $1062-1076$.

Kolendo J., Mobilité sociale $=$ J. Kolendo, La perception et l'appréciation d'un status social: le cas de primi pili, [in:] E. Frézouls (Hg.), La mobilité sociale dans le monde romain. Actes du colloque de Strasbourg (novembre 1988), Strasbourg 1992, S. 161-173.

Légions $=$ Les légions de Rome sous le Haut-Empire (Actes du Congrès de Lyon, 17-19 septembre, 1998), Hg. Y. Le Bohec, C. Wolff, I-II, Lyon 2000.

Łajtar A., Żelazowski J., Le nuove iscrizioni provenienti da Scodra (Albania) e il nuovo v(ir) e(gregius) ducenarius, Zeitschrift für Papyrologie und Epigraphik 192, 2014, S. 273-283.

Millar F., A Study of Cassius Dio, Oxford 1964.

Moga V., Piso I., Drîmbărean M., L'aigle de la legio XIII Gemina, Acta Musei Napocensis 43-44, 2006-2007, S. 177-184.

Mrozewicz L., Legio I Italica i legio XX Valeria victrix: Związki wzajemne, Eos 74, 1986, S. 303-308.

Mrozewicz L., Victoria Aug(usta) Panthea Sanctissima, Zeitschrift für Papyrologie und Epigraphik 57, 1984, S. 181-184.

Petiș I.-A., The centurions' promotion system, Acta Musei Napocensis 41-42, 2004-2005, S. $125-140$.

Pflaum H.-G., Jupiter Depulsor, [in:] Mélanges Isidor Lévy, Paris 1955, S. 445-460. 
Piso I., Les listes de centurions de Potaissa et la participation des legions daciques à la guerre parthique de Caracalla, [in:] A. Tomas (ed.), Ad fines Imperii Romani. Studia Thaddaeo Sarnowski septuagenario ab amicis, collegis discipulisque dedicata, Warsaw 2015, S. 81-88.

Roman Frontier Studies $1989=$ Roman Frontier Studies 1989. Proceedings of the XVth International Congress of Roman Frontier Studies, Exeter 1991.

Sarnowski T., Die Ziegelstempel aus Novae. I. Systematik und Typologie, Archeologia 34, 1983 (1985), S. 17-61.

Sarnowski T., Römische Militärziegel von der südwestlichen Krim. Probleme der Produktionstätigkeit und Produktionsorte, Archeologia (Warszawa) LVI 2005, S. 141-152.

Sarnowski T., The headquarters building of the legionary fortress at Novae (Lower Moesia), [in:] Roman Frontier Studies 1989, S. 303-307.

Thomasson B.E., LP = Laterculi praesidum I, Göteborg 1984.

Thomasson B.E., LP = Laterculi praesidum I, Göteborg 1984.

Zwicky H., Zur Verwendung des Militärs in der Verwaltung der römischen Kaiserzeit, Zürich 1944. 
